\title{
Open Education
}

Open Education Consortium

\section{Source}

Open Education Consortium. What is Open Education?

Open education encompasses resources, tools and practices that employ a framework of open sharing to improve educational access and effectiveness worldwide. 